\title{
A Comparative Analysis of Reference Architectures for Healthcare in the Ambient Assisted Living Domain
}

\author{
Lina Garcés \\ Department of Computer Systems \\ University of São Paulo - USP \\ São Carlos, Brazil \\ linamgr@icmc.usp.br
}

\author{
Apostolos Ampatzoglou, Paris Avgeriou \\ Department of Mathematics and \\ Computer Science \\ University of Groningen - RUG \\ Groningen, The Netherlands \\ a.ampatzoglou@rug.nl,paris@cs.rug.nl
}

\author{
Elisa Yumi Nakagawa \\ Department of Computer Systems \\ University of São Paulo - USP \\ São Carlos, Brazil \\ elisa@icmc.usp.br
}

\begin{abstract}
Population aging has brought important challenges at social, economical, and healthcare issues, mainly due to the increasing number of people that need intensive care. Ambient Assisted Living (AAL) systems that aim at assisting people in their health-related activities have emerged. In another perspective, Reference Architectures (RAs) are a special type of software architecture that promotes reuse of design expertise and facilitates the development, standardization, and evolution of software systems. During the last years, important RAs for AAL systems have been created. However, there is a lack of studies that compare RAs for healthcare systems in AAL domain, making the selection among RAs a rather difficult task. In this paper, we present a comparative analysis and evaluate the completeness of that healthcare RAs. Specifically, we intend to offer: (i) a guide to select the most complete and adequate RA for software projects; and (ii) to describe research directions to improve existing and future RAs for that systems.
\end{abstract}

Keywords-Healthcare; Reference Architecture; Interoperability; Ambient Assisted Living;

\section{INTRODUCTION}

Ambient Assisted Living (AAL) has become an increasingly important, interdisciplinary research topic for both the medical and the technological research communities [1]. AAL refers to concepts, products, and services aiming at enhancing several aspects of people's quality of life, including autonomy/independence, comfort, safety, security, and health in all stages of their life [1]. Moreover, software systems for AAL includes concepts, methodologies, and technologies from eHealth aiming at the integration of assistive technologies and services into existing systems of nursery, healthcare and eldercare providers [2].

Considering the relevance of AAL software systems in the society, and the diversity of application domains and technologies that AAL embrace, researchers, practitioners, and organizations (i.e., IT and healthcare providers) have advised the importance of creating heterogeneous, interoperable, open, and reusable platforms and standards for AAL domain. For this reason, several Reference Architectures (RAs) have been proposed, supported, principally, by the European Commission, under the FP6 and FP7 research calls ${ }^{1}$. In short, a RA is a generic type of software architecture that achieves well-recognized knowledge of specific domains, which promotes reuse of design expertise and facilitates the development, standardization, and evolution of software systems. Moreover, a RA captures the essence of architectures of similar systems, and provides a common lexicon and taxonomy, a common architectural vision, and the modularization and the complementary context [3]. Using RA facilitates the development of interoperable AAL software systems in a cheaper, faster and more efficient way.

However, despite the importance of RA for AAL systems, to the best of our knowledge the state of the art lacks of a comprehensive and in-depth analysis of RAs for Healthcare in the AAL domain. The aforementioned lack makes the selection of RAs for developing, standardizing, and evolving Healthcare software systems a rather difficult task. In this context, the main objective of this paper is to present the results obtained from studing the completeness of available RAs proposed for Healthcare systems in the AAL domain. To achieve this goal, we used the RAModel [4] which facilitates the understanding of the RAs, and the identification of elements (i.e., domain, application, infrastructure, and crosscutting elements) that are missing in existing RAs.

The remainder of this paper is organized as follows. Section II presents the methods. Section III exposes the related works. Section IV reports the analysis on RAs for healthcare systems in AAL domain. Discussions about our analysis are presented in Section V. Finally, Section VI presents our conclusions.

\section{RESEARCH METHODS}

In order to obtain the RAs proposed in the AAL domain, we conducted a systematic literature review following the guidelines proposed by Kitchenham and Charters [5]. As first step, we established the following search string:

\footnotetext{
("Ambient Assisted Living" OR "ambient assisted" OR "ambient assistance" OR "assisted environments" OR "assistive environments" OR "assisted environment" $O R$ "assistive environment" $O R$ " $A A L$ environment" $O R$ " $A A L$ environments" $O R$

${ }^{1}$ http://cordis.europa.eu/fp7/
} 
“independent living" OR "assisted life" OR "intelligent living” OR "pervasive healthcare" OR "pervasive care") AND ("Reference Architecture" OR "reference architectures" OR "reference model" OR "reference models")

Next, we adapted the string to seven well-known digital libraries proposed by Dyba et al. [6] and Kitchenham and Charters [5], as the most relevant sources in computer science area, i.e., ACM Digital Library, IEEE Xplore, SpringerLink, ScienceDirect, Engineering Village, Scopus, and Web of Science. Next, we selected primary studies based on reading of title, abstract, and keywords. Included studies were read in full with the aim to include those studies strictly related to our research objective (i.e., identification of RAs for the AAL domain). As a result of this process we discovered 11 reference architectures proposed for the AAL domain. Finally, we classified 5 of these reference architectures as related to Healthcare software systems, and that are presented in Table I.

Moreover, in order to analyse the completeness of reference architectures for healthcare systems, we used the RAModel [4], a reference model for reference architectures, providing information on possibly all elements (and their relationships) that could be contained in reference architectures, independently from application domains or purpose of such architectures [4]. RAModel defined the following five groups of elements:

- Domain elements: Elements related to specific information of the space of human action in the real world which impact or restrict software systems, i.e., legislations, standards, regulations, quality attributes, and system compliance;

- Application elements: Elements that provide a good understanding of the reference architecture, its capabilities and limitations. It also contains elements related to the business rules (or functionalities) that can be present in software systems built from the reference architecture, i.e., constraints, domain data, functional requirements, goals \& needs, limitations, and risks ;

- Infrastructure elements: Elements that can be used to build the software systems based on the reference architecture. These elements are responsible for enabling these systems to automate, for instance, processes, activities, and tasks of a given domain, i.e., best practices $\&$ guidelines, general structure, hardware elements, and software elements; and

- Crosscutting elements: It aggregates a set of elements that are usually spread across and/or tangled with elements of other three groups (domain, application, and infrastructure), such as, communication (that can be defined as internal and external) in the software systems built from the reference architecture, as well as the domain terminology and decisions are present in a spread and tangled way when describing other groups.
Table I

REFERENCE ARCHITECTURES FOR HEALTHCARE SYSTEMS

\begin{tabular}{llll}
\hline ID & Reference & Title & Year \\
\hline RA1 & Liu et al. [9] & $\begin{array}{l}\text { Reference Architecture of Intelligent } \\
\text { Appliances for the Elderly }\end{array}$ & 2005 \\
\hline RA2 & $\begin{array}{l}\text { Hietala et al. } \\
{[10]}\end{array}$ & $\begin{array}{l}\text { FeelGood - Ecosystem of PHR based } \\
\text { products and services }\end{array}$ & 2009 \\
\hline RA3 & $\begin{array}{l}\text { Kameas and } \\
\text { Calemis } \\
\text { [[12]) }\end{array}$ & Pervasive Systems in Health Care & 2010 \\
\hline RA4 & $\begin{array}{l}\text { Wartena et } \\
\text { al. [13] }\end{array}$ & $\begin{array}{l}\text { Continua: The Reference Architecture } \\
\text { of a Personal Telehealth Ecosystem }\end{array}$ & 2010 \\
\hline RA5 & $\begin{array}{l}\text { Tuomainen } \\
\text { and } \\
\text { Mikkanen } \\
\text { ([14]) }\end{array}$ & $\begin{array}{l}\text { Reference architecture of application } \\
\text { services for personal wellbeing infor- } \\
\text { mation management. }\end{array}$ & 2011 \\
\hline & & & \\
\hline
\end{tabular}

\section{RELATED WORK}

To the best of our knowledge, there are two studies assessing AAL platforms, frameworks, and software systems [7], [8]. Antonino et al. [7] present an evaluation of several AAL platforms (i.e., Alhambra, Hydra, OASIS, OpenAAL, PERSONA, and UniversAAL), with respect to quality attributes relevant for architecture (i.e., reliability, security, maintainability, efficiency, and safety) and their characteristics, through semi-structured interviews. Memon et al. [8] provide a literature survey on AAL frameworks, systems and platforms to identify the essential aspects of such systems and investigate the critical issues from design, technology, quality of service, and user experience point of view.

Our study differs from the aforementioned studies in two aspects. We present (i) an evaluation of the completeness of reference architectures with a focus on its elements; and (ii) a more focused study, in the sense that it is strictly related to Healthcare software systems and not in the entire AAL domain.

\section{REFERENCE ARCHITECTURES FOR HEALTHCARE IN THE AAL DOMAIN}

In this section we present the identified application domains addressed by the reference architectures. Selection of such domains was made based on classification proposed by the AALIANCE project [1]. Moreover, we describe the elements defined by each reference architecture, and analyse their level of completeness.

\section{A. Application domains}

Reference architectures for healthcare software systems have been established for four types of applications, as listed in Table II.

- Personal care: Software systems, sometimes involving assistance robots, to support people in carrying out activities of daily life such as, taking drugs, dressing and undressing, and personal hygiene. Personal care 
Table II

APPLICATION DOMAINS

\begin{tabular}{|c|c|c|c|c|c|}
\hline Application domain & $\begin{array}{l}\text { RA } \\
\mathbf{1}\end{array}$ & \begin{tabular}{l|}
$\mathbf{R A}$ \\
2
\end{tabular} & $\begin{array}{l}\text { RA } \\
3\end{array}$ & $\begin{array}{l}\text { RA } \\
4\end{array}$ & \\
\hline Personal care & $\mathrm{X}$ & & $\mathrm{X}$ & $\mathrm{X}$ & $\mathrm{X}$ \\
\hline Person centered health management & & $\mathrm{X}$ & $\mathrm{X}$ & $\mathrm{X}$ & $\mathrm{X}$ \\
\hline $\begin{array}{l}\text { Telemonitoring and self-management } \\
\text { of chronic diseases }\end{array}$ & & & $\mathrm{X}$ & $\mathrm{X}$ & \\
\hline Personal activity management & & & $\mathrm{X}$ & $\mathrm{X}$ & \\
\hline
\end{tabular}

systems can be developed based on the knowledge presented in reference architectures RA1, RA3, RA4, and RA5.

- Person centered health management: Software systems that allow the access to medical records (e.g., containing current treatment, chronic diseases, allergies, or medications), through the use of mobile devices. Such systems empowers the person with relevant knowledge and with online support allowing him or her to take more responsibility for their own health. Results of our study show that RA2, RA3, RA4, and RA5 can guide the establishment of this type of applications.

- Telemonitoring and self-management of chronic diseases: Software systems that monitor the physiological parameters of people and control their health (in most cases is not in an invasive manner). To achieve this goal, such systems can use wearable sensors, advanced signal processing techniques and network systems. This information can be provided remotely to users, their families and clinicians in order to make known constantly the health condition of the subject, to make an exact diagnosis, to identify the correct therapies and to intervene at the right time. These systems are supported by RA3 and RA4.

- Personal activity management: Software systems that monitor persons' activities of daily life (ADL) aiming to provide information about a physical or mental condition. These systems can signal cognitive decline or to prevent incidents. Personal activity management systems can be developed using reference architectures RA3 and RA4.

\section{B. Analysis of Completeness}

Table III presents the RAModel [4] elements (as described in Section II), in order to understand which information is contained, and which is missing, in the definitions of RAs proposed for Healthcare software systems into AAL domain. In the remainder of this section we present a brief discussion of the elements defined by each reference architecture.

1) RA1 - SISARL: This reference architecture aims to be a foundation for the identification, definition and standardization of internal and external interfaces of SISARL (Sensor Information Systems/Services for Active Retires and
Table III

RAMODEL ELEMENTS DEFINED BY EACH REFERENCE ARCHITECTURE

\begin{tabular}{|c|c|c|c|c|c|c|}
\hline Group & Element & $\begin{array}{l}\mathbf{R A} \\
\mathbf{1}\end{array}$ & $\begin{array}{l}\mathbf{R A} \\
\mathbf{2}\end{array}$ & $\begin{array}{l}\mathbf{R A} \\
\mathbf{3}\end{array}$ & $\begin{array}{l}\text { RA } \\
4\end{array}$ & $\begin{array}{l}\text { RA } \\
\mathbf{5}\end{array}$ \\
\hline \multirow{3}{*}{ Domain } & $\begin{array}{l}\text { Legislations, standards, and } \\
\text { regulations }\end{array}$ & & $\mathrm{X}$ & & $\mathrm{X}$ & $\mathrm{X}$ \\
\hline & Quality Attributes & & $\mathrm{X}$ & & $\mathrm{X}$ & $\mathrm{X}$ \\
\hline & System compliance & & & & $\mathrm{X}$ & \\
\hline \multirow{7}{*}{$\begin{array}{l}\text { Applica- } \\
\text { tion }\end{array}$} & Constraints & $\mathrm{X}$ & $\mathrm{X}$ & & $\mathrm{X}$ & \\
\hline & Domain data & $\mathrm{X}$ & $\mathrm{X}$ & & $\mathrm{X}$ & $\mathrm{X}$ \\
\hline & Functional requirements & & $\mathrm{X}$ & & $\mathrm{X}$ & $\mathrm{X}$ \\
\hline & Goal \& needs & & $\mathrm{X}$ & & $\mathrm{X}$ & $\mathrm{X}$ \\
\hline & Limitations & & $\mathrm{X}$ & & & $\mathrm{X}$ \\
\hline & Risks & & $\mathrm{X}$ & & & \\
\hline & Scope & & $\mathrm{X}$ & $\mathrm{X}$ & $\mathrm{X}$ & $\mathrm{X}$ \\
\hline \multirow{4}{*}{$\begin{array}{l}\text { Infrastruc- } \\
\text { ture }\end{array}$} & Best practices and guidelines & & $\mathrm{X}$ & & $\mathrm{X}$ & \\
\hline & General structure & $\mathrm{X}$ & $\mathrm{X}$ & $\mathrm{X}$ & $\mathrm{X}$ & $\mathrm{X}$ \\
\hline & Hardware elements & $\mathrm{X}$ & $\mathrm{X}$ & $\mathrm{X}$ & $\mathrm{X}$ & \\
\hline & Software elements & $\mathrm{X}$ & $\mathrm{X}$ & $\mathrm{X}$ & $\mathrm{X}$ & $\mathrm{X}$ \\
\hline \multirow{4}{*}{$\begin{array}{l}\text { Crosscu- } \\
\text { tting } \\
\text { elements }\end{array}$} & Decisions & & $\mathrm{X}$ & & $\mathrm{X}$ & \\
\hline & Domain terminology & $\mathrm{X}$ & $\mathrm{X}$ & $\mathrm{X}$ & $\mathrm{X}$ & \\
\hline & External communication & $\mathrm{X}$ & $\mathrm{X}$ & & $\mathrm{X}$ & $\mathrm{X}$ \\
\hline & Internal communication & $\mathrm{X}$ & $\mathrm{X}$ & & $\mathrm{X}$ & \\
\hline
\end{tabular}

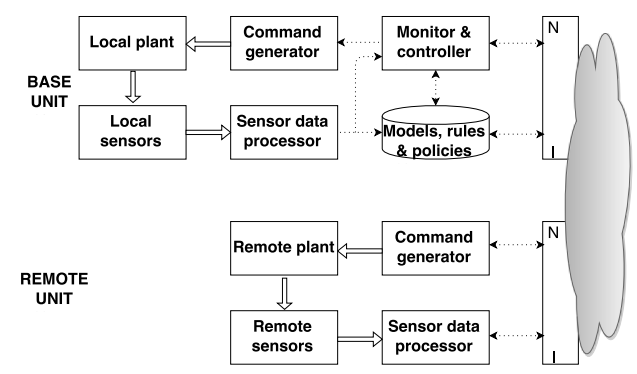

Figure 1. SISARL reference architecture. Adapted from [9]

Assisted Living) appliances and systems [9]. Moreover, it is oriented to Personal care applications, as presented in Table II.

Regarding application elements, SISARL reference architecture defines constraints, principally in regards the design of appliances with different purpose. As important domain data, RA1 defines information from local plant, local sensors, sensor data processor, command generator, monitor and controller, and communication units.

SISARL defined its general structure, and its hardware and software elements. Appliance general structure defined by SISARL is composed by two layers, i.e., base unit and remote unit (See Figure 1). Units are composed principally by the following software and hardware components [9]:

(i) Sensor data processor that encodes sensor data streams;

(ii) Monitor and controller is the key component, that uses local sensor data to estimate current plant state, and data from communication unit to obtain the desired state of the 
plant; (iii) Local database that stores user data (i.e., heartbeat record, and medication record) and rules and policies governing the behavior and usage of the appliance; and (iv) Communication unit allowing exchange of information with the outside world (i.e., Cellphones).

Finally, SISARL details crosscutting elements, i.e., domain terminology, external communication through network interfaces, and internal communication centralized by the monitor and controller component.

Drawbacks: SISARL reference architecture does not describe domain elements hindering the understanding of laws, standards, regulations that SISARL systems must address. Moreover, the lack of quality attributes makes difficult the architectural design and validation of such systems. Additionally, it does not offer a clear understanding about the limitation of its use, the set of systems that could be developed using it, the risk of use it, and the functional requirements that are important when software systems are evaluated by stakeholders. It lacks of guidelines defining well-experimented practices to develop SISARL systems, and it was not found documentation about rationale obtained during the reference architecture development.

2) RA2 - FeelGood: Aims to guide the service evolution of interoperable PHR (Personal Health Records) services in order to allow a better comparability among different solutions. Concerning to our study, FeelGood is considered the most complete reference architecture, since provides documentation on majority of elements .

FeelGood defines a set of standards composed by HL7, DICOM, XPHR, and SNOMED-CT [10]. Also, it uses knowledge stored in more general reference architectures as SOAP, and OASIS. As important quality attributes for PHR systems, FeelGood establishes: security, confidentiality, authenticity, reliability, interoperability, compatibility, maintainability, and scalability.

FeelGood general structure includes service components and interfaces covering different PHR services and ecosystems (See Figure 2). PHR service client and server are the most important components. PHR service client is a software application (or browser) running on a PC or a mobile device. Client can additionally be connected to measurement devices and specific monitoring servers for exchanging data. At client part, iPAN, iWAN and iXHR interfaces [10] maps to the reference topology described in Continua Guidelines [11]. The client part may also be connected to a separate storage media (iPO interface). The server part may exchange information with other applications and services [10].

Drawbacks: FeelGood lacks of means (e.g., supporting frameworks or tools) that allow verify that PHR systems are created following standards or legislations defined by this RA.

3) RA3 - Pervasive Systems in Healthcare: This reference architecture aims to guide the development of pervasive healthcare systems to monitoring vital signs using ECG,

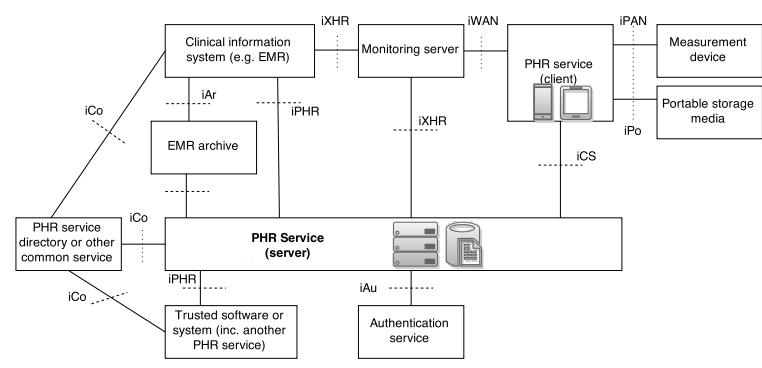

Figure 2. FeelGood reference architecture. Adapted from [10]

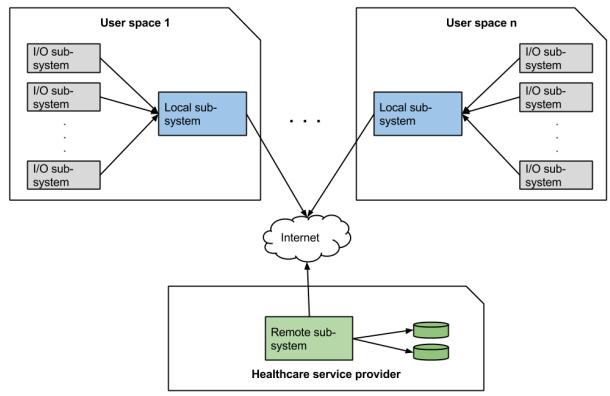

Figure 3. High-level view of the reference architecture for pervasive healthcare systems.

HRM, BPM, and SpO2. As showed in Table II, RA3 intends to cover the four application domains, and defines, mainly, infrastructure elements, as presented in Table III.

RA3 is composed by three subsystems [12] as showed in Figure 3: (i) Input-Output subsystems, that use sensory input of varying data types. This input is provided by sensors attached either to the space or to the user, by wearable devices or by specific healthcare devices that retrieve specific measurements; (ii) Local subsystem, that locally collects sensory and other information and provides it to the healthcare system. The local subsystem may appear in the form of a single computer or as a small computing device like a PDA; and (iii) Remote subsystem, that receives the data collected by the local subsystems and either does some kind of processing on data, or stores it into health medical records. Usually the remote subsystem resides on Healthcare service providers, capable of providing scientific monitoring of the data and checking whether the patient is in a critical condition or not in order to provide immediate support.

Drawbacks: It does not define domain elements, i.e., quality attributes, system compliance, legislations, standards, and regulations. Neither, in this RA is established application elements such as, constraints, domain data, functional requirements, goal and needs, limitations, and risks. This RA also lacks of best practices and guidelines definition for its use and instantiation. Additionally, there is an absence of decisions, rationale, and external/internal communication description. Moreover, it was developed based on technical analysis of previous pervasive healthcare systems, 
without considering users requirements, standards, reference models, ontologies, or another information source; and because of this, RA3 only defines its general structure, hardware/software elements, and scope (i.e., pervasive healthcare systems) in a high abstract way. Furthermore, it is not possible to develop interoperable systems based on this RA, due to it does not establish interfaces for its sub-systems.

4) RA4 - Continua: Continua Health Alliance is an international not-for-profit industry organization enabling end-toend, plug-and-play connectivity of devices and services for personal health management and healthcare delivery. Its mission is to empower information-driven health management and facilitate the incorporation of health and wellness into the day-to-day lives of consumers [11]. Moreover, it intends to cover all application domains defined in Table II.

Continua's general structure is defined by reference device classes (See Figure 4). Such reference device classes are: (i) PAN and LAN at first tier; (ii) Application Hosting Device at second tier; (iii) WAN device at third tier, and (iv) Health Reporting Device at last tier. Additionally, four principal interfaces that connects the bottom tiers with its upper tier are defined (i.e., PAN, LAN, WAN, and HRN interfaces) [13]. Continua describes standards for its interfaces. For PAN interface it were selected the next standards: (i) Bluetooth Health Device Profile for wireless communication; (ii) USB Personal Healthcare Devices for wired communication; (iii) ISO/IEEE 11073 Personal Health Device family to enable data format interoperability; and (iv) ZigBee Health Care Profile for the transport level. Regarding WAN interface it was selected the Integrating the Healthcare Enterprise (IHE) Cross-Enterprise Document REliable Interchage (XDR) profile as a means to establish the communication among WAN and HRN devices [13]. Moreover, the HL7 Personal Health Monitoring (PHM) Report document format was chosen to ensure consistent data encoding.

Continua considers the following quality characteristics as important for health management systems, i.e., compatibility, interoperability, security, authenticity, confidentiality, non-repudiation, integrity, reliability, and availability.

Furthermore, Continua defines a homogeneous security framework that helps to: (i) ensure compliance with legislation, (ii) increase usability, (iii) allow seamless integration of security mechanisms, and (iv) support end-to-end identity management, integrity and confidentiality, as well as origin authentication and non-repudiation.

Drawbacks: Continua documentation lacks of information about its risks and limitations at using either as a whole or parts of it. This information is considered important due to Continua was defined in a high-level of abstraction, making difficult its instantiation at developing concrete systems.

5) RA5 - Coper: Aims to orient the development of software systems for information management in different wellbeing-related services, such as, PHR, electronic government services, and personal time and content management

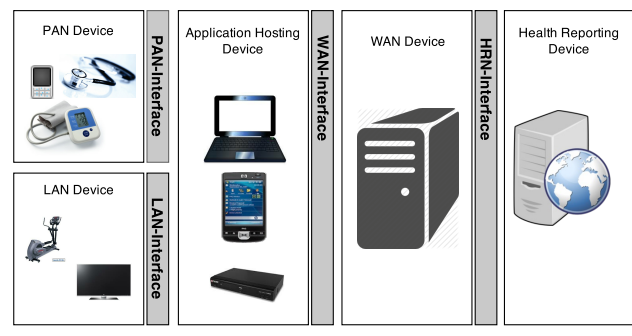

Figure 4. Continua reference architecture. Adapted from [13]
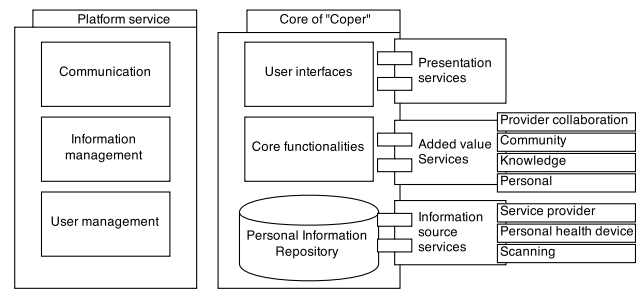

Figure 5. Coper reference architecture. Adapted from [14]

tools.

Coper reference architecture defines the following standards: HL7 CDA implementation guides, HL7 continuity of care document specifications, and IHE Exchange of personal health record content profile. Moreover, interoperability and changeability are considered as important quality attributes for wellbeing information management systems.

Coper general structure is based on Service-Oriented Architecture (SOA). Services are classified according to functional, platform, information or interactivity requirements (See Figure 5).

Drawbacks: Coper lacks of techniques to evaluate system compliance to legislation, standards, and regulations. Additionally, it does not specify its constraints and risks at using this RA. At infrastructure level, there is a lack of best practices and guidelines that orient its use. Internal communication among its components, hardware elements, decisions, and domain terminology were not defined.

\section{DISCUSSION}

Despite the existence of consolidated reference architectures for healthcare systems in the AAL domain, several application domains, previously identified by [1], must be addressed by current or future RAs: (i) Support for care givers and care organizations (e.g., reasoning systems on all available health-related data); (ii) Biorobotic systems (e.g., operational machine or exoskeleton-like machines for rehabilitation); and (iii) Prevention of diseases and injuries at work.

Based on the results obtained from the completeness analysis, we can conclude that the most complete and consolidated RAs are FeelGood and Continua. It is important to highlight that both RAs have involved participants from 
governmental organizations, healthcare providers, research centers, IT organizations, and community, from different countries. Moreover, one of main objectives for creating both RAs was to offer guidelines for developing interoperable systems. In this context, we believe that the inception phase at developing a RA must be supported by interdisciplinary teams, in order to establish the domain and application elements for the RA.

Current RAs do not define guidelines to overtake issues related to the security of health records (e.g., confidentiality, integrity, non-repudiation, and authenticity). In this scenario, existing and future RAs must define approaches to ensure security requirements of healthcare systems.

Complete RAs can be constructed based on more abstract ones. This was observed from FeelGood, which reused knowledge contained in Continua, SOAP, and OASIS reference architectures. In this context, future RAs could reuse specific elements from RAs presented in this study. To achieve this, a comprehensive and detailed documentation of all existing RAs elements must be provided (e.g., following RAModel elements definition).

We observed that application domains addressed by available RAs have the final user as the most important stakeholder (i.e., aged or disabled persons). However, existing RAs do not consider important quality attributes, such as usability, usefulness and satisfaction level, at developing healthcare software systems. In this perspective, more efforts to establish approaches that guide the creation of $\mathrm{HCI}$ (Human Computer Interaction) or HRI (Human Robotics Interaction) interfaces are needed.

\section{CONCLUSION}

Reference architectures have gained more attention from practitioners and academics, since they allow to understand the knowledge obtained of software development in specific domains. In this work, we detail the ARs available for healthcare systems in AAL domain. The main contribution of this work is to present an overview of each RA and to establish drawbacks. Moreover, results of this work can be used by software practitioners or researchers to select the better RA for its projects.

\section{ACKNOWLEDGMENT}

This work is supported by Brazilian funding agencies Capes/Nuffic (Grant N.: 034/12) and FAPESP (Grants N.: 2014/02244-7 and 2013/20317-9).

\section{REFERENCES}

[1] Broek, G.V.D., Cavallo, F., Wehrmann, C.: AALIANCE Ambient Assisted Living Roadmap. IOS Press, Amsterdam, The Netherlands (2010).

[2] Buchmayr, M., Kurschl, W.: A survey on situation-aware ambient intelligence systems. Journal of Ambient Intelligence and Humanized Computing, 2(3),175-183 (2011).
[3] Cloutier, R., Muller, G., Verma, D., Nilchiani, R., Hole, E., Bone, M.: The Concept of Reference Architectures. Syst. Eng.. 13(1),14-27 (2010)

[4] Nakagawa, E.Y., Oquendo,F., Becker, M.: RAModel: A Reference Model for Reference Architectures. In: Proceedings of the 2012 Joint Working IEEE/IFIP Conference on Software Architecture and European Conference on Software Architecture, WICSA-ECSA '12, pp. 297-301. IEEE Computer Society, Washington, DC, USA (2012).

[5] Kitchenham, B.A., Charters, S.: Guidelines for performing systematic literature reviews in software engineering. Technical report, Keele University and Durham University, UK (2007).

[6] Dyba, T., Dingsoyr, T., Hanssen, G.K.: Applying systematic reviews to diverse study types: An experience report. In: First International Symposium on Empirical Software Engineering and Measurement, ESEM, pp. 225-234 (2007).

[7] Antonino, P.O., Schneider, D., Hofmann, C., Nakagawa, E.Y.: Evaluation of AAL platforms according to architecture-based quality attributes. Lecture Notes in Computer Science (including subseries Lecture Notes in Artificial Intelligence and Lecture Notes in Bioinformatics), 7040 LNCS, pp. 264-274 (2011).

[8] Memon, M., Wagner, S.R., Pedersen, C.F., Beevi, F.H.A., Hansen, F.O.: Ambient assisted living healthcare frameworks, platforms, standards, and quality attributes. Sensors, 14, 43124341 (2014).

[9] Liu, J.W.S., Wang, B.Y., Liao, H.Y., Huang, C.Y., Shih, C.S., Kuo, T.W., Pang, A.C.: Reference architecture of intelligent appliances for the elderly. In: 18th International Conference on Systems Engineering, pp. 447-455 (2005).

[10] Hietala, H., Ikonen, V., Korhonen, I., Lahteenmaki, K., Maksimainen, A., Pakarinen, V., Parkka, J., Saranummi,N.: Feelgood - ecosystem of phr based products and services. Research report VTT-R-07000-09, VTT Technical Research Centre of Finland.,Tampere, Finland (2009).

[11] Continua design guidelines, version 2013. Continua Health Alliance, pp. 403 (2013).

[12] Kameas, A., and Calemis, I.: Pervasive systems in health care. In: Nakashima, H., Aghajan, H., Augusto, J.C. (eds.) Handbook of Ambient Intelligence and Smart Environments, pp. 315-346. Springer US (2010).

[13] Wartena, F., Muskens, J., Schmitt, L., Petkovic, M.: Continua: The reference architecture of a personal telehealth ecosystem. In: 12th IEEE International Conference on e-Health Networking Applications and Services (Healthcom), pp. 1-6 (2010).

[14] Tuomainen, M., Mikkanen, J. Reference architecture of application services for personal wellbeing information management. In: Studies in Health Technology and Informatics. User Centred Networked Health Care, pp. 98-102. IOS Press Ebooks. (2011).

[15] Fagerberg, G., Kung, A., Wichert, R., Tazari, M., Jean-Bart, B., Bauer, G.: Platforms for AAL applications. In: Lukowicz, P., Kunze, K., Kortuem, G. (eds.) Smart Sensing and Context. LNCS, vol. 6446, pp.177-201. Springer Berlin Heidelberg (2010). 